\title{
Health benefits of tai chi: potential mechanisms of action
}

Keywords: Tai Chi, mind-body, mechanism of action, mental health, exercise, physical health, psychological, aging population, health system, therapy, chronic disease, relaxed mind, promote health

\section{Introduction}

Optimal human movement requires continuous integration of afferent (incoming) and efferent (outgoing) nervous system impulses. Humans cannot flow through the environment safely unless they concurrently take in information with their senses (e.g. visual, auditory, somatosensory and tactile information), integrate these ascending impulses, and plan and execute the next motor action. Indeed, one does not move until one first has the inspiration (thought) to move. Tai Chi (TC) is a mind and body exercise that provides a solid foundation for all functional movement (e.g. activities of daily living, work, play and sport applications). From its roots as a martial art, TC practice fosters the development of both efficient and effective movement. ${ }^{1} \mathrm{TC}$ was first described in the scientific literature in $1981 .^{2} \mathrm{TC}$ emphasized an alert, yet relaxed mind, and a strong, yet supple body. Traditional Chinese Medicine distinguished TC as a holistic exercise with strong preventative elements. It was considered ideal for balancing one's mind and body, creating an overall sense of peace and harmony and naturally inspiring longevity. ${ }^{3}$ The Chinese proclaimed TC as a spiritual exercise beneficial for mental health (psychological wellbeing) and physical health by decreasing blood pressure, providing mild aerobic exercise, improving flexibility (particularly spinal mobility) and promoting the conservation of energy (Qi) through efficient and effective (i.e. functional) movements. ${ }^{2,3}$

Modern medicine describes TC is a meditative movement therapy ${ }^{4}$ that fosters functional movement patterns addressing the strength, endurance, mobility and balance impairments frequently seen in the aging population. ${ }^{1-5}$ Today, the health benefits of TC are renowned and address many of the mortality and morbidity factors associated with lifestyle-related noncommunicable diseases and conditions (e.g. osteoarthritis, heart disease, cognitive impairment and dementia, chronic obstructive pulmonary disease, stroke) seen it the $21^{\text {st }}$ century. $^{6-9}$ The rising burden of chronic disease poses a challenge for the health system and requires innovative approaches to optimize aging and wellness. ${ }^{6-9}$ Community-based and individual practice of TC holds promise as a health promotion and wellness exercise as well as an intervention for a variety of chronic health conditions afflicting older adults. ${ }^{1-9}$

But how exactly does TC work to achieve these many health benefits; what is its mechanism of action? A linear rise in psychoneuroimmunology (PNI) publications $(\mathrm{N}=1104$ articles since 2000) reflects the growing suspicion that a disease-oriented biomedical model is insufficient in addressing the chronic health conditions facing aging adults in the $21^{\text {st }}$ century..$^{10}$ The field of PNI seeks to understand the mechanisms by which both drug and non-drug interventions such as TC, yoga and mindfulness-based meditation promote health. ${ }^{10,11}$ The intent of this paper is to evaluate Chinese mind-body principles and guidelines of TC practice in an effort to comprehend how TC is able to impact multiple dimensions of wellness within the human experience (Figure 1).
Volume 2 Issue 5 - 2018

\section{Kristine M Hallisy}

Department of Family Medicine and Community Health, University of WI-Madison School of Medicine and Public Health, USA

Correspondence: Kristine M Hallisy, PT, DSc, Department of Family Medicine and Community Health, University of WI-Madison School of Medicine and Public Health, Doctor of Physical Therapy Program, USA, Tel 608 263-6744, Fax 608 2627809,Email hallisy@pt.wisc.edu

Received: July 18, 2018 | Published: September 05, 2018

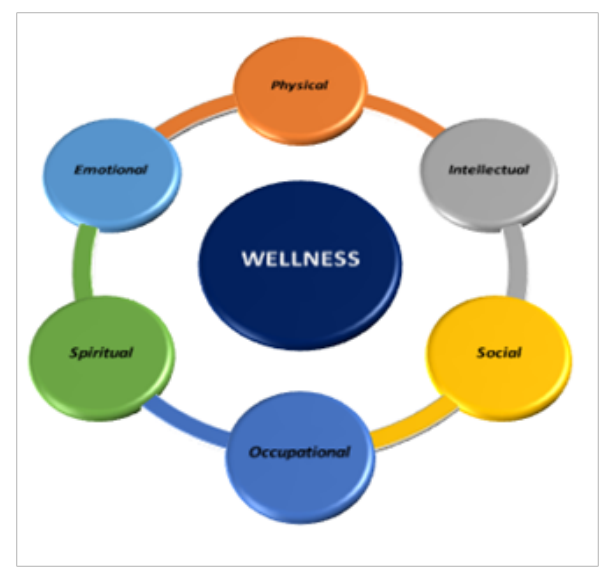

Figure I Tai chi impacts dimensions of wellness within the human experience.

\section{Chinese mind-body principles}

Three Chinese mind-body principles (Table 1 ) and seven guidelines (Figure 2) for TC practice inform how TC is instructed and practiced. The mind-body principles (centering, effective action, TC energetics) and guidelines for TC practice are integral to all styles of TC and may explain how TC imparts its health benefits. ${ }^{12}$ Centering directs us to "get in touch with our body and our surroundings." 12 TC begins with a few moments (to minutes) of quiet, focused attention into the present moment. This stillness prepares the mind and body for the flowing movement of TC. The mindful practice of TC creates effective action that is rooted in the feet, powered by the legs, guided by the torso and expressed in the hands. ${ }^{12}$ Body mechanics and spontaneous action are hallmarks of the effective action of TC. The third mind-body principle, TC energetics, promotes sensory awareness and balanced, relaxed flowing movement. ${ }^{12}$

\section{Guidelines of TC practice}

Mindfulness - strive to focus on the present moment. Mindfulness is the intentional and non-judgmental focus of one's attention on the 
emotions, thoughts, sensations and actions currently taking place. ${ }^{13}$ A simple review of the scientific literature (PubMed "mindfulness benefits, systematic reviews, last 5 years") yielded $n=51$ articles touting the physiological and psychological benefits of mindfulness. Meditative practices impart general health benefits as well as improvement from depression, mental illness, anxiety, pain and other psychological stressors. ${ }^{14}$ There is evidence of decreased levels of cortisol, epinephrine and norepinephrine (stress-related hormones) with mind-body interventions like TC, yoga and meditation. Moreover, PNI-based interventions are also associated with reductions in inflammatory processes and levels of pro-inflammatory cytokines in cancer, HIV, depression, anxiety, wound healing, sleep disorder, cardiovascular diseases and fibromyalgia. ${ }^{15}$

Table I Chinese Mind-Body Principles - definition, Chinese tenet, English translation and application

\begin{tabular}{|c|c|c|}
\hline Mind-Body principle & Chinese tenet & English components (Translation) \\
\hline Centering Mindfulness & Be still as a mountain, move like a great river. & $\begin{array}{l}\text { Bring attention into the present moment. } \\
\text { Feel the movement in your body as you breathe. } \\
\text { Keep body uptight and naturally aligned } \\
\text { Bring awareness into hands, fingers and feet. } \\
\text { Notice the sounds, sights and smells around you. }\end{array}$ \\
\hline $\begin{array}{l}\text { Effective Action } \\
\text { Body Mechanics }\end{array}$ & $\begin{array}{l}\text { Tai chi is rooted in the feet, powered by the legs, } \\
\text { directed by the torso and expressed in the hands. }\end{array}$ & $\begin{array}{l}\text { Movement is from the ground up with proper postural } \\
\text { alignment and body mechanics. } \\
\text { With the body relaxed and aligned, spontaneous } \\
\text { movements are naturally circular. } \\
\text { Efficient and effective movement allows us to move } \\
\text { (flow) around obstacles. }\end{array}$ \\
\hline $\begin{array}{l}\text { Tai Chi Energetics } \\
\text { Sensory Awareness }\end{array}$ & $\begin{array}{l}\text { Relaxed and rooted to the earth, the body moves } \\
\text { effortlessly like a floating cloud (moving like a string } \\
\text { of pearls). }\end{array}$ & $\begin{array}{l}\text { Heavy: Knees, tailbone and elbows are relaxed, and feel } \\
\text { heavier. } \\
\text { Light: Head, eyes, fingers are light and uplifted fostering } \\
\text { the flow of Qi (energy) into the hands. }\end{array}$ \\
\hline Mindfulness & \begin{tabular}{c|cc} 
Postural & Breath & Active \\
Alignment & Awareness & Relaxation
\end{tabular} & \begin{tabular}{c|c} 
Slow & Weight \\
Movement & Separation
\end{tabular} \\
\hline
\end{tabular}

Figure 2 Guidelines for TC Practice.

Postural Alignment - maintain the body in an upright posture. TC posture (Figure 3) reminds us to maintain an upright spinal posture as we move throughout our day. The flexed knee posture readies us for action while supporting the natural primary (thoracic kyphosis) and secondary (cervical and lumbar lordotic) curves of the spine. Based on engineering (tensegrity) principles, the spinal curves contribute to the structural integrity and balance of the spine, enhancing strength and protecting us from injury. ${ }^{16,17}$ Use of the powerful muscles of triple extension in the lower extremity (e.g. hip extensors, quadriceps and plantar flexors) along with the 3-dimensional mobility of the hip joint further protects the spine as we use our extremities to complete the many tasks of our day.

Breathe Awareness - relaxed, natural diaphragmatic breathing. At the center of all meditative movement therapies (e.g.TC, qigong, yoga) is breath awareness. Breathing is essential to life, and to be thankful for each breath is essential to happiness. Indeed, when a baby first emerges from the womb, its first action of life is a deep and nourishing diaphragmatic breath. The stress of everyday life frequently steals away the body's natural tendency to perform these natural cleansing breaths. TC practice reminds us to slow down and inhale deeply to nourish and exhale completely to cleanse every cell in our being. During the practice of TC, deep diaphragmatic breathing is integrated into body motions to achieve a harmonious balance between body and mind and to facilitate the flow of internal energy (Qi). ${ }^{9}$ Depending on style of TC (e.g. Yang, Chen, Sun, Wu or Wu-Hao) and underlying health of the participant, TC is a mild-to moderate aerobic exercise appropriate for a variety of cardiopulmonary diseases, and can likewise by used in the general community as a preventative health measure. $^{3-9}$ 


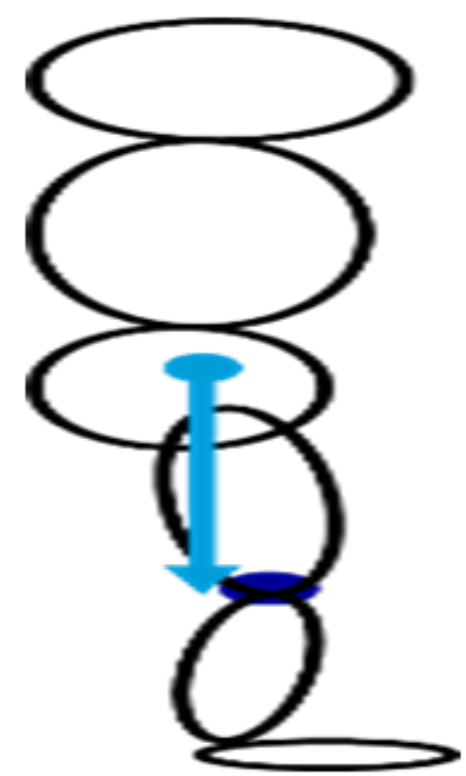

Figure 3 TC posture: Rooted in the feet, powered by the legs (quadriceps), directed by the torso and expressed in the hands.

Active Relaxation - a state of relaxed inner stillness while in motion. Long believed to be the most difficult, active relaxation is the center point of the seven guidelines for TC practice (Figure 2). While all seven guidelines are simultaneously performed in $\mathrm{TC}$, it is active relaxation that reminds us to integrate inner calmness while simultaneously taking on safe and effective physical action. Motor learning and motor control theory tells us that while mental practice and physical practice alone both enhance movement, but the combination of the two (dual tasking) is most effective. ${ }^{18-20}$

Slow Movement - builds strength and endurance. ${ }^{7-21}$ While slow movement is more difficult it allows the nervous system time to create optimal balance between agonistic and antagonistic muscles. It affords the brain ample time to integrate our joints, and to craft the precise action we wish to accomplish. Slow movement, when repeated, and done in a relaxed way, prepares the nervous system for more dynamic, rapid, and even, ballistic functional activities. The slow movement of TC is the roadmap to motor efficiency and power, and precisely why many martial artists practice $\mathrm{TC}$ on the way to mastering their more explosive martial arts practices.

Weight Separation - enhances dynamic control of the center of mass (postural control and balance). Unless we learn to separate our weight, we will always be stuck in one place. Weight separation is what allows us to step forward, step backward and side-step the troubles in our lives. Mindfully separating and accepting one's weight as we walk is fundamental to safe movement in our environment. The weight separation and stepping transitions of TC practice readily satisfies principle \#1 of Sherrington's best practice exercise guidelines for falls prevention. TC provides mid- to high- level balance challenge by 1) moving the center of mass over the base of support, 2) reducing the base of support from double limb to single limb support and 3) decreasing the need for upper limb support in standing. ${ }^{22} \mathrm{TC}$ is a readily adaptable exercise accessible to a wide variety of ages and skill levels. Traditional free-standing TC can be modified to seated, standing with side support, and or walker support versions. ${ }^{23,24} \mathrm{TC}$ can be used in individual or group community-based settings to promote weight-separation, kinesthesia (static postural awareness) and dynamic postural control. ${ }^{1-23}$

Integrated Movement form the Core - proximal stability for distal mobility. All the Grandmasters of TC tell us to return to the core (dantian) when we are having trouble in our TC practice. The dantians (e.g. upper [forehead between the eyebrow], middle [at the level of the heart] and lower [below the navel]) are important points of reference in TC and other practices (e.g. qigong, Reiki, traditional Chinese medicine). The lower dantian, also known as the qi (energy) center is particularly important as it is the focal point of breathing as well as the body's center of gravity. ${ }^{25}$ All TC (and indeed human) movements are initiated from the core. In rehabilitation medicine, this phenomenon is known as proximal stability for distal mobility. ${ }^{26-28}$ Core muscles provide stability that allows generation of force and motion in the lower extremities, and a stable base of support for upper extremity function. ${ }^{28}$

\section{Benefits of TC exercise}

The benefits of exercise have a historical precedence established long before they were touted by $13^{\text {th }}$ century Chinese TC practitioners. Ancient physicians including Susruta of India $(600 \mathrm{BC})$ and Hippocrates (480-370 BC) prescribed exercise to promote health. ${ }^{29}$ In modern times, the health benefits of exercise cannot be denied by the health care system. Physical exercise is the only intervention that consistently attenuates age-related declines in physical function. ${ }^{30}$ Hutson and McFarlane (2016) noted the general health and fitness benefits of TC to be balance, aerobic capacity, strength, well-being, sleep, and flexibility. ${ }^{7}$ Both these family physicians felt there was robust evidence to recommendation TC to their patients. Furthermore, key features of TC practice include: mindfulness (awareness of the present moment), imagery (images used as mental learning strategy), structural alignment (postures and movements that are effective and efficient), flexibility and relaxation, strength and balance, natural (diaphragmatic) breathing, social support (positive interactions within a community) and integration of body, mind and spirit (holistic health practice).$^{5-31}$ As with any physical activity, the health benefits of TC are inevitably associated with practice duration, intensity, level of mastery, and commitment.

\section{Summary}

TC is a meditative movement therapy that fosters optimal functional movement patterns that can be applied to all human movements. TC has the capacity to go far beyond its widely accepted use for balance and falls prevention and has benefits for a wide variety of health conditions. ${ }^{5}$ Its preventative qualities can be traced back to its original Chinese tenets.TC is a readily adaptable exercise accessible to a wide variety of ages and skill levels. While TC is considered a mind (intellectual) and body (physical) exercise, it can impact all domains of wellness within the human experience (Figure 3). It has the potential to mindfully impact our ability to move efficiently and effectively in our personal, social and occupational environments. This in turn, provides human beings with emotional stability expanding our sense of purpose and providing meaning to our lives (spiritual wellness). While the opening statement to this paper - Transforming society by optimizing movement to improve the human experience," is the vision statement of the American Physical Therapy Association (APTA), it is also precisely why Tai Chi (TC) works: "Movement is life. Life is good, therefore, movement must be good." 


\section{Acknowledgements}

None.

\section{Conflict of interest}

The author declares there is no conflict of interest.

\section{References}

1. Hackney ME, Wolf SL. Impact of Tai Chi Chu'an practice on balance and mobility in older adults: an integrative review of 20 years of research. $J$ Geriatr Phys Ther. 2014;37(3):127-35.

2. Koh TC. Tai Chi Chuan. Am J Chin Med. 1981;9(1):15-22.

3. Zhou DH. Preventive geriatrics: an overview from traditional Chinese medicine. Am J Chin Med. 1982;10(4):32-39.

4. Larkey L, Jahnke R, Entier J, et al. Meditative movement as a category of exercise: implications for research. J Phys Act Health. 2009;6(2):230-38.

5. Hallisy KM. Tai chi beyond balance and fall prevention: Health benefits and its potential role in combatting social isolation in the aging population. Curr Geri Rep. 2018;7(1):37-48.

6. Dean E, Greig A, Murphy S, et al. Raising the priority of lifestyle-related noncommunicable diseases in physical therapy curricula (perspective). Phys Ther. 2016;96(7):940-848.

7. Hutson P, McFarlane B. Health benefits of tai chi: clinical review. Can Fam Physician. 2016;62(11):881-890.

8. Solloway MR, Taylor SL, Shekelle PG, et al. An evidence map of the effect of Tai Chi on health outcomes. Syst Rev. 2016;5(1):126.

9. Lan C, Chen SY, Lai JS, et al. Tai chi chuan in medicine and health promotion. Evid Based Complement Alternat Med. 2013;2013:502131.

10. Kelley KW. To boldly go where no one has gone before. Brain Behav Immun. 2017;14:1-8.

11. Kelley GA. Kelley KS. Meditative Movement Therapies and HealthRelated Quality-of-Life in Adults: A Systematic Review of MetaAnalyses. PLoS One. 2015;8;10(6):e0129181.

12. Yu T, Johnson J. Tai Chi Fundamentals for Health Care Professionals. Taos, NM: Uncharted Country Publishing, 1999.

13. Kabat-Zinn J. Full catastrophe living: using the wisdom of your body and mind to face stress, pain, and illness. New York: Delta; 1990. p. 76-77.

14. Acevedo BP, Pospos S, Lavretsky H. The Neural Mechanisms of Meditative Practices: Novel Approaches for Healthy Aging. Curr Behav Neurosci Rep. 2016;3(4):328-339.

15. Moraes LJ, Miranda MB, Loures LF, et al. A systematic review of psychoneuroimmunology-based interventions. Psychol Health Med. 2018;23(6):635-652.
16. Myers T. Spatial Medicine - A call to 'arms." J Bodyw Mov Ther. 2014;18(1)94-98.

17. Yip SK. Optimal postural alignment using tai chi principles. (C) (on-line), 2008.

18. Eaves DL, Riach M, Holmes PS, et al. Motor imagery during action observation: A brief review of evidence, theory and future research opportunities. Front Neurosci. 2016;21;10:514.

19. Filgueiras A, Quintas Conde EF, Hall CR. The neural basis of kinesthetic and visual imagery in sports: an ALE meta-analysis. Brain Imaging Behav. 2017;19.

20. Ghai S, Ghai I, Effenberg AO. Effects of dual tasks and dual-task training on postural stability: a systematic review and meta-analysis. Clin Interv Aging. 2017;23;12:557-577.

21. Watanabe Y, Madarame H, Ogasawara R, et al. Effect of very low-intensity resistance training with slow movement on muscle size and strength in healthy older adults. Clin Physiol Funct Imaging. 2014;34(6):463-470.

22. Sherrington $\mathrm{C}$, Tiedemann A, Fairhall N, et al. Exercise to prevent falls in older adults: an updated meta-analysis and best practice recommendations. N S W Public Health Bull. 2011;22(3-4):78-83.

23. Yu T, Hallisy KH. Tai Chi Fundamentals ${ }^{\circledR}$ The Adapted Program with Optional Side Support, Walker Support, and Seated Versions [Book]. Taos NM: Uncharted Country Publishing, 2015.

24. Yu T. Tai Chi Fundamentals ${ }^{\circledR}$ The Adapted Program with Optional Side Support, Walker Support, and Seated Versions [DVD]. Taos NM: Uncharted Country Publishing, 2015.

25. Dantian (dan tien). From Wikipedia, the free encyclopedia (on-line). 2018.

26. Oliver GD, Washington JK, Barfield JW, et al. Quantitative analysis of proximal and distal kinetic chain musculature during dynamic exercise. $J$ Strength Cond Res. 2018;32(6):1545-1553.

27. Gottschall JS, Mills J, Hastings B. Integration core exercises elicit greater muscle activation than isolation exercises. J Strength Cond Res. 2013;27(3):590-596.

28. Rivera CE. Core and Lumbopelvic Stabilization in Runners. Phys Med Rehabil Clin N Am. 2016;27(1):319-337.

29. Tipton CM. The history of "Exercise Is Medicine" in ancient civilizations. Adv Physiol Educ. 2014;38(2):109-117.

30. Buford TW, Hsu FC, Brinkley TE, et al. Genetic influences on exerciseinduced changes in physical function among mobility-limited older adults. Physiol Genomics. 2014;1;46(5):149-158.

31. Wayne PM. The Harvard Medical School Guide to tai chi: 12 weeks to a healthy body, strong heart and sharp mind. Boston MA: Shambhala Publications, Inc. 2012. p. 352. 